\title{
Электрические и фотоэлектрические свойства структур металл-диэлектрик-полупроводник на основе Si с наночастицами Au на границе раздела диэлектрик/полупроводник
}

\author{
(C) М.Н. Коряжкина, С.В. Тихов, О.Н. Горшков, А.П. Касаткин, И.Н. Антонов \\ Национальный исследовательский Нижегородский государственный университет им. Н.И. Лобачевского, \\ 603095 Нижний Новгород, Россия \\ E-mail: mahavenok@mail.ru
}

(Получена 27 апреля 2016 г. Принята к печати 10 мая 2016 г.)

Показано, что формирование наночастиц $\mathrm{Au}$ на границе раздела диэлектрик/кремний в структурах с высокой плотностью поверхностных состояний приводит к смещению энергии закрепления уровня Ферми на этой границе раздела к потолку валентной зоны кремния и увеличению плотности поверхностных состояний при энергиях, близких к уровню Ферми. На кривых фоточувствительности конденсаторной фотоэдс при этом появляется полоса с максимумом при 0.85 эВ, которая объясняется фотоэмиссией электронов и состояний, примыкающих к потолку валентной зоны кремния и образованных наночастиц $\mathrm{Au}$.

\section{1. Введение}

Формирование наночастиц (англ.: nanoparticle) металлов в диэлектрических слоях используется с целью изучения и использования плазмонных эффектов [1], а также для улучшения характеристик флеш-памяти [2] и элементов памяти на основе резистивного переключения $[3,4]$, которое наблюдается как в структурах металл-диэлектрик-металл (МДМ) [3], так и в структурах металл-диэлектрик-полупроводник (МДП) [4,5]. В настоящей работе изучены электрические и фотоэлектрические свойства МДП-структур на основе $\mathrm{Si}$ c наночастицами $\mathrm{Au}(n p-\mathrm{Au})$ на границе раздела (ГР) диэлектрик/полупроводник (Д/П).

\section{2. Методика эксперимента}

МДП-структуры $\mathrm{Au} / \mathrm{Zr} / \mathrm{SiO}_{x} / \mathrm{np}-\mathrm{Au} / \mathrm{Si}$ и $\mathrm{Au} / \mathrm{Zr} / \mathrm{YSZ} / \mathrm{np}$ $\mathrm{Au} / \mathrm{Si}$ формировались методом магнетронного распыления на установках MagSputt-3G-2 и 2g1-1g2-eb4-th1 на подложках монокристаллических пластин $\mathrm{Si}$ (100) КЭФ-4.5 (концентрация электронов $n_{0}=1.8 \cdot 10^{15} \mathrm{~cm}^{-3}$ ), покрытых химическим методом (последовательной обработкой поверхности $\mathrm{Si}$ вначале в плавиковой кислоте, а затем в смеси серной кислоты с перекисью водорода) туннельно-тонким слоем $\mathrm{SiO}_{2}$, на который осаждался слой $\mathrm{Au}$ с эффективной толщиной в 1 нм при температуре подложки $200^{\circ} \mathrm{C}$. В качестве диэлектрика использовались пленки $\mathrm{SiO}_{x}$ и стабилизированного оксидом иттрия (12 мол\%) диоксида циркония (англ.: yttria (12 mol\%) stabilized zirconia) (YSZ). Толщина пленок составляла 40 нм. Пленки осаждались при температуре подложки $300^{\circ} \mathrm{C}$. Подобные пленки использовались ранее при формировании проявляющих резистивное переключение МДМ-структур на основе $\mathrm{SiO}_{x}[6]$ и МДП-структур c YSZ в качестве диэлектрика [5]. Полученные таким образом структуры кратковременно отжигались в атмосфере аргона при температуре $450^{\circ} \mathrm{C}$. После отжига на поверхность слоя диэлектрика осаждались полупрозрачные для освещения верхние электроды из $\mathrm{Au}$ площадью $S=1.0 \cdot 10^{-2} \mathrm{~cm}^{2}$ и толщиной 15 нм (с подслоем из $\mathrm{Zr}$ толщиной 3 нм для улучшения адгезии). Подобное формирование $n p-A u$ описано в [7]. Контрольными образцами являлись структуры без $n p$-Аu.

Измерялись зависимости от напряжения $U$ и частоты $f$ малосигнальной емкости $C$ и проводимости $G$ в параллельной эквивалентной схеме замещения конденсатора [8] в интервале частот $f=10^{3}-2 \cdot 10^{6}$ Гц и температур $T=200-500 \mathrm{~K}$ при скорости развертки по напряжению $77 \mathrm{MB} / \mathrm{c}$ в темноте и на свету (использовалась галогенная лампа мощностью 50 Вт). Измерения проводились на анализаторе параметров полупроводниковых приборов Agilent B1500A. Проводились также измерения конденсаторной фотоэдс насыщения на одиночных импульсах длительностью $\sim 10$ мс с использованием фотовспышки типа ФИЛ-107 (поток квантов $\sim 5.0 \cdot 10^{19}$ фот/см ${ }^{2}$ с с в максимуме спектрального распределения 0.5 мкм) по методике [9] с использованием запоминающего осциллографа GDS-71022 и кривых спектральной чувствительности фотоэдс $S_{\mathrm{ph}}$ по методике из работы [10]. Напряжение на структуре соответствовало потенциалу верхнего электрода относительно потенциала подложки.

\section{3. Экспериментальные результаты}

Все исследованные структуры характеризовались относительно высокой плотностью поверхностных состояний (ПС) и частотной дисперсией $C U$ - и $G U$-характеристик. На рис. 1 приведены зависимости емкости и приведенной к круговой частоте проводимости $G / \omega$ от напряжения на разных частотах тестирующего сигнала для МДП-структуры $\mathrm{Au} / \mathrm{Zr} / \mathrm{YSZ} / n p-\mathrm{Au} / \mathrm{Si}$. 


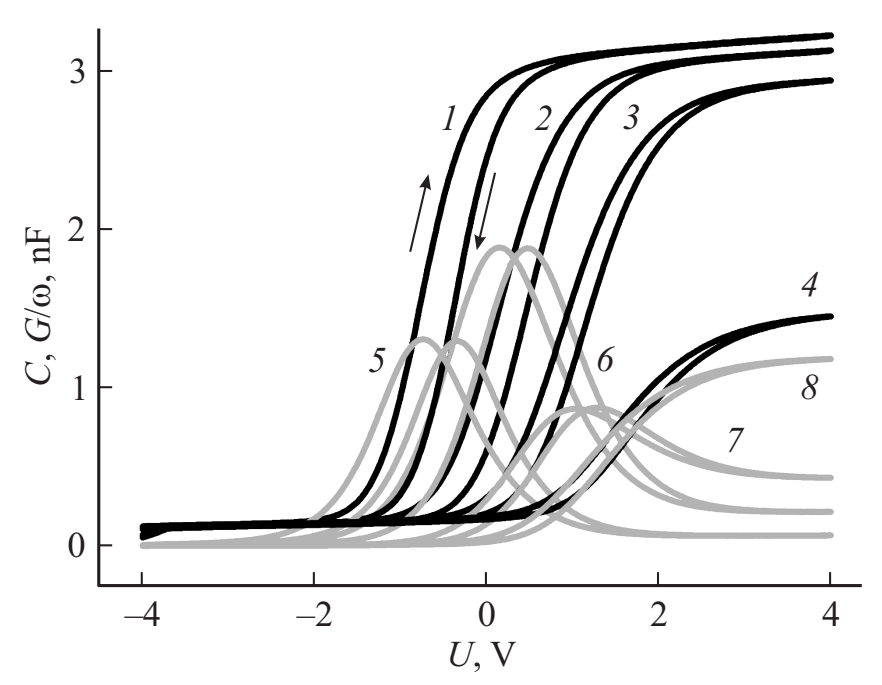

Рис. 1. Зависимости $C(1-4)$ и $G / \omega(5-8)$ от $U$ для МДП-структуры $\mathrm{Au} / \mathrm{Zr} / \mathrm{YSZ} / n p-\mathrm{Au} / \mathrm{Si}$, полученные на частоте тестирующего сигнала $f$, кГц: $1,5-1 ; 2,6-10 ; 3,7-10^{2}$; $4,8-10^{3}$. Стрелками показано направление развертки по напряжению.

Значительная частотная дисперсия была связана с влиянием ПС, так как емкость полупроводника в области ее модуляции напряжением возрастала с уменьшением частоты [8]. С ростом частоты уменьшалась максимальная емкость МДП-структуры, которая обычно связывается с емкостью слоя диэлектрика. Поэтому использование этих характеристик для определения основных параметров МДП-структуры (изгиба зон в полупроводнике, характера энергетического спектра плотности ПС и параметров диэлектрика) было существенно затруднено. В связи с этим для установления влияния $n p-\mathrm{Au}$ на изгиб зон в полупроводнике и энергетический спектр ПС были использованы измерения сильносигнальной импульсной фотоэдс и частотной зависимости емкости и приведенной к круговой частоте тестирующего сигнала проводимости $G / \omega$ при изменении температуры.

Согласно данным работы [11], в случае одиночного приповерхностного барьера в полупроводнике, когда при больших потоках квантов (обычно $10^{18}-10^{20}$ фот./см · с) происходит насыщение фотоэдс, ее величина становится равна темновому поверхностному потенциалу полупроводника.

Для образцов с $n p-\mathrm{Au}$ конденсаторная фотоэдс насыщения, которая была равна величине начального темнового потенциала поверхности $\mathrm{Si} \varphi_{s}$, составляла $0.35 \mathrm{~B}$ для структур $\mathrm{Au} / \mathrm{Zr} / \mathrm{YSZ} / n p-\mathrm{Au} / \mathrm{Si}$ и 0.40 В для структур $\mathrm{Au} / \mathrm{Zr} / \mathrm{SiO}_{x} / n p-\mathrm{Au} / \mathrm{Si}$. Так как равновесный уровень Ферми в объеме $\mathrm{Si}$ равен $F=E_{c}-0.25$ эВ, эти значения соответствовали инверсии поверхности Si. В контрольных образцах значения $\varphi_{s}$ составляли $0.23 \mathrm{~B}$ для структур c YSZ и $0.25 \mathrm{~B}$ для структур с $\mathrm{SiO}_{x}$, что отвечало истощению поверхности $\mathrm{Si}$.
На рис. 2 показаны частотные зависимости $C$ и $G / \omega$ для МДП-структуры $\mathrm{Au} / \mathrm{Zr} / \mathrm{YSZ} / n p-\mathrm{Au} / \mathrm{Si}$ при $U=0$ и разных температурах.

Качественно аналогичные зависимости были получены для всех исследованных структур. Для анализа приведенных на рис. 2 данных были использованы соотношения [8]

$$
\begin{gathered}
C_{I}=C_{D} C_{\min } /\left(C_{D}-C_{\min }\right), \\
C_{I}=\left(q \varepsilon_{0} \varepsilon_{s} n_{0} / 2\left|V_{S I}\right|\right)^{1 / 2},
\end{gathered}
$$

где $C_{D}$ - емкость диэлектрика, $q-$ заряд электрона, $\varepsilon_{0}-$ абсолютная диэлектрическая проницаемость вакуума, $\varepsilon_{s}$ - относительная диэлектрическая проницаемость диэлектрика, $V_{S I}$ - предельное значение потенциала, падающего на слое обеднения полупроводника, граничащего с инверсным слоем. Минимальная емкость $C_{\min }$ МДП-структур на частотных зависимостях (см. например, на рис. 2) определялась в структурах с $n p-\mathrm{Au}$ инверсионной емкостью $C_{I}$ полупроводника в соответствии с выражениями (1) и (2), а в структурах без $n p$-Аu емкость $C_{\min }$ оказывалась выше. Частотные зависимости для структур с $n p-\mathrm{Au}$ были сдвинуты в область более высоких частот по сравнению со случаем структур без nр-Аu при одинаковых температурах. Эти данные согласуются с результатами, полученными при измерении фотоэдс насыщения. Во всех структурах при относительно низких частотах $(1-10$ кГц) достигалась емкость диэлектрика $C_{D}$, поскольку на этих частотах преобладала емкость ПС, как в случае достаточно высоких температур при отсутствии освещения, так и в случае температуры $300 \mathrm{~K}$ при наличии освещения

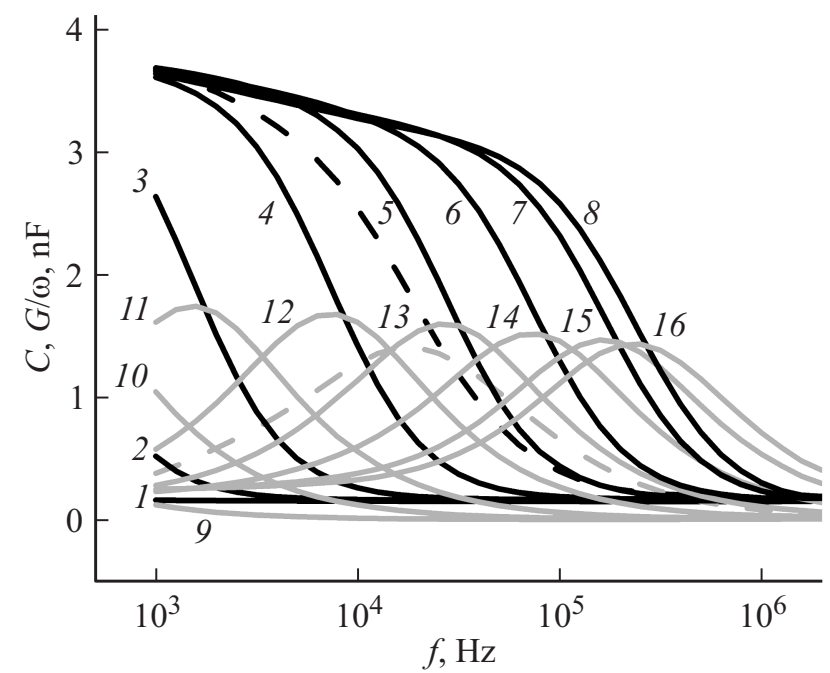

Рис. 2. Частотные зависимости $C(1-8)$ и $G / \omega(9-16)$ для МДП-структуры $\mathrm{Au} / \mathrm{Zr} / \mathrm{YSZ} / n p-\mathrm{Au} / \mathrm{Si}$, полученные в темноте, при $U=0$ и разных температурах $T, \mathrm{~K}: 1,9-300$; $2,10-335 ; 3,11-365 ; 4,12-400 ; 5,13-435$; $6,14-463 ; 7,15-490 ; 8,16-500$. Пунктирной линией показаны частотные зависимости $C$ и $G / \omega$, полученные при освещении при $300 \mathrm{~K}$. 


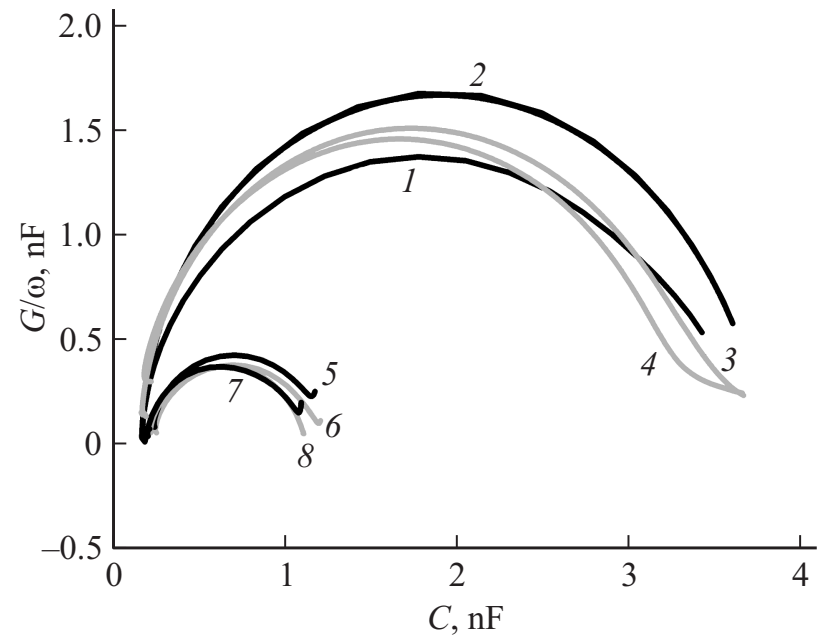

Рис. 3. Зависимости $G / \omega$ от $C$ для МДП-структур $\mathrm{Au} / \mathrm{Zr} / \mathrm{YSZ} / n p-\mathrm{Au} / \mathrm{Si}(1-4)$ и $\mathrm{Au} / \mathrm{Zr} / \mathrm{SiO}_{x} / n p-\mathrm{Au} / \mathrm{Si}(5-8)$, измеренные при комнатной температуре и освещении $(1,8)$ и в темноте при температуре $T, \mathrm{~K}: 2-400,3-435,4-490$, $5-425,6-385,7-360$.

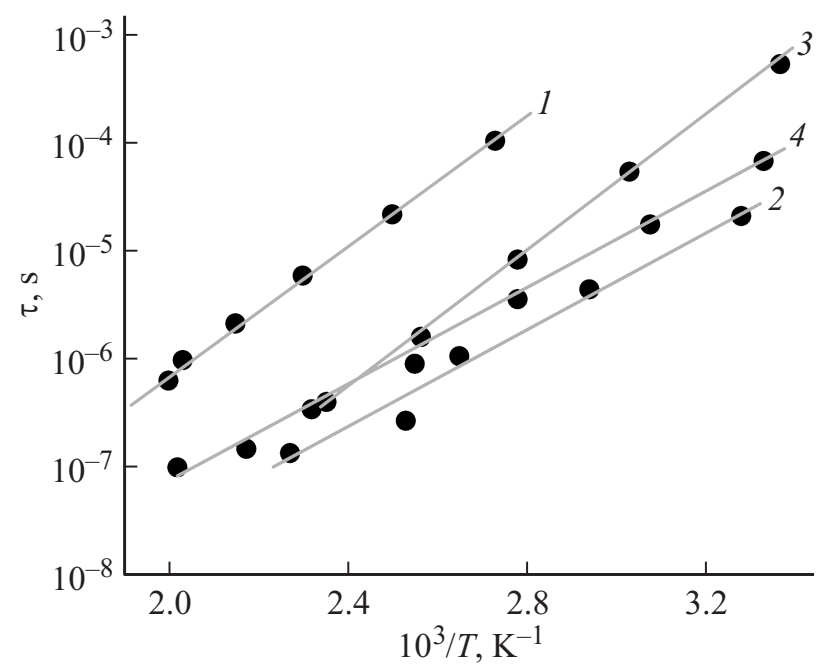

Рис. 4. Зависимости $\tau$ от $10^{3} / T$ для МДП-структур $\mathrm{Au} / \mathrm{Zr} / \mathrm{YSZ} / n p-\mathrm{Au} / \mathrm{Si}(1), \mathrm{Au} / \mathrm{Zr} / \mathrm{YSZ} / \mathrm{Si}$ (2), $\mathrm{Au} / \mathrm{Zr} / \mathrm{SiO}_{x} /$ $n p-\mathrm{Au} / \mathrm{Si}(3)$ и $\mathrm{Au} / \mathrm{Zr} / \mathrm{SiO}_{x} / \mathrm{Si}(4)$.

(пунктирная линия на рис. 2). Анализ полученных частотных зависимостей с помощью построения диаграмм Коула-Коула [12] (рис. 3) показал, что эти частотные зависимости не могут быть описаны релаксацией носителей на моноэнергетическом поверхностном уровне, так как эти диаграммы не представляли собой полуокружности. Таким образом, энергетическое распределение ПС было квазинепрерывным.

Параметры ПС были определены, используя эквивалентную схему области пространственного заряда (ОПЗ) полупроводника при параллельном соединении емкости ОПЗ и связанных с захватом электронов поверхностными состояниями емкости и проводимости [8].
При этом потери в диэлектрике не учитывались ввиду их малости. Величины плотности ПС $N_{s s}$ были определены по значениям максимума зависимости от частоты приведенной к круговой частоте проводимости $(G / \omega)_{\max }$ (см. рис. 2) в соответствии с выражением [8]

$$
(C / \omega)_{\max } \approx 0.4 q^{2} N_{s s}
$$

Величины $N_{s s}$ слабо зависели от температуры, напряжения и освещения для всех исследованных образцов. Поэтому можно утверждать, что уровень Ферми на поверхности Si был закреплен в результате высокой плотности донорных и акцепторных состояний в точке электронейтральности [13] с энергией $E_{N}=q \varphi_{s}+E_{c}-F$.

Наличие $n p-\mathrm{Au}$ приводило к сдвигу точки электронейтральности ближе к потолку валентной зоны (от $E_{c}-0.45$ эВ до $E_{c}-0.60$ эВ для МДП-структур $\mathrm{Au} / \mathrm{Zr} / \mathrm{YSZ} / n p-\mathrm{Au} / \mathrm{Si}$ и от $E_{c}-0.45$ эВ до $E_{c}-0.65$ эВ для МДП-структур $\left.\mathrm{Au} / \mathrm{Zr} / \mathrm{SiO}_{x} / n p-\mathrm{Au} / \mathrm{Si}\right)$ в результате акцепторного действия $\mathrm{Au}$, что проявлялось в росте отрицательного заряда на поверхности Si. Подтверждением этого, наряду с фотоэлектрическими измерениями, были зависимости эффективного времени релаксации $\tau$ поверхностных состояний от обратной температуры, показанные на рис. 4.

Время релаксации $\tau$ определялось по значению частоты $f_{\text {max }}$, соответствующему максимуму частотной зависимости $(G / \omega)_{\max }$ по формуле $\tau=1 / 2 \pi f_{\max }$ [8]. Как следовало из данных рис. 4, приведенные на нем зависимости имели линейный вид, что позволяло определить из них значения энергии активации $\Delta E(\tau \sim \exp [-\Delta E / k T])$, которые оказались равны $0.59(1), 0.63(3), 0.45(2,4)$ и практически совпадали со значениями $q \varphi_{s}+E_{c}-F$ при комнатной температуре, определенными по насыщению фотоэдс для каждой из исследованных МДП-структур. Так как эти энергии активации практически совпадали со значениями энергии закрепления (пиннинга) уровня Ферми на поверхности $\mathrm{Si}$, можно считать, что определяемые энергии активации соответствовали не захвату электронов поверхностными состояниями, а эмиссии из ПС (барьер для эмиссии электронов на 0.25-0.40 эВ выше, чем для захвата). В этом случае время эмиссии $\tau$, согласно [14], было равно

$$
\tau=e_{n}^{1}=\left(\sigma_{n} \cdot u_{T n} \cdot N_{c}\right)^{-1} \exp \left[\left(E_{c}-E_{t}\right) / k T\right],
$$

где $e_{n}-$ скорость эмиссии электронов из ловушки, $\sigma_{n}-$ сечение захвата электрона ловушкой, $u_{T n}$ - тепловая скорость электрона, $N_{c}$ - плотность состояний в $c$-зоне, $E_{t}$ - энергия поверхностного уровня, совпадающего с уровнем Ферми на поверхности Si. Из равенства (4), в частности, следовало, что при возрастании температуры время релаксации резко уменьшалось. Логарифмирование равенства (4) давало выражение

$$
\ln \left(\tau \cdot T^{2}\right)=-\ln \left(\sigma_{n} \cdot b_{n}\right)+\left(E_{c}-E_{t}\right) / k T,
$$

из которого можно определить величину $\sigma_{n}$. Константа $b_{n}$ для $\mathrm{Si}$ равна $6.6 \cdot 10^{21} \mathrm{~cm}^{-2} \cdot \mathrm{c}^{-1} \cdot \mathrm{K}^{-2}[14]$. Из 
Параметры диэлектриков и малосигнальной эквивалентной схемы МДП-структур

\begin{tabular}{l|l|l|c|c|c}
\hline \multicolumn{1}{c|}{ Структура } & $\varphi_{s}, \mathrm{~B}$ & $E_{N}$ & $E_{a},{ }^{\mathrm{B}}$ & $\sigma_{n}, \mathrm{~cm}^{2}$ & $N_{s s}, \mathrm{~cm}^{-2}{ }^{-1} \mathrm{~B}^{-1}$ \\
\hline $\mathrm{Au} / \mathrm{Zr} / \mathrm{YSZ} / n p-\mathrm{Au} / \mathrm{Si}$ & 0.35 & $E_{c}-0.60$ эB & 0.59 & & $3.8 \cdot 10^{12}$ \\
$\mathrm{Au} / \mathrm{Zr} / \mathrm{YSZ} / \mathrm{Si}$ & 0.23 & $E_{c}-0.45$ эB & 0.45 & $1.5 \cdot 10^{-16}$ & $1.8 \cdot 10^{12}$ \\
$\mathrm{Au} / \mathrm{Zr} / \mathrm{SiO}_{2} / n p-\mathrm{Au} / \mathrm{Si}$ & 0.4 & $E_{c}-0.65$ эB & 0.63 & & $8.9 \cdot 10^{11}$ \\
$\mathrm{Au} / \mathrm{Zr} / \mathrm{SiO}_{2} / \mathrm{Si}$ & 0.25 & $E_{c}-0.45 \mathrm{\jmath} \mathrm{B}$ & 0.45 & $1.5 \cdot 10^{-14}$ & $7.3 \cdot 10^{11}$
\end{tabular}

соотношения (5), полагая, что $\sigma_{n}$ не зависит от температуры, получили, что значение $\sigma_{n}$ равно $1.5 \cdot 10^{-16} \mathrm{~cm}^{2}$ для структур с YSZ и $1.5 \cdot 10^{-14} \mathrm{~cm}^{2}$ для структур с $\mathrm{SiO}_{x}$. Увеличение значений концентрации ПС в точке электронейтральности, обусловленное формированием $n p-A u$, было определено из соотношения (3). Это увеличение происходило от $1.8 \cdot 10^{12}$ до $3.8 \cdot 10^{12} \mathrm{~cm}^{-2} \cdot$ эВ $^{-1}$ в случае структур с YSZ и от $7.3 \cdot 10^{11}$ до $8.9 \cdot 10^{11} \mathrm{~cm}^{-2} \cdot$ эВ $\mathrm{B}^{-1}$ в случае структур с $\mathrm{SiO}_{x}$.

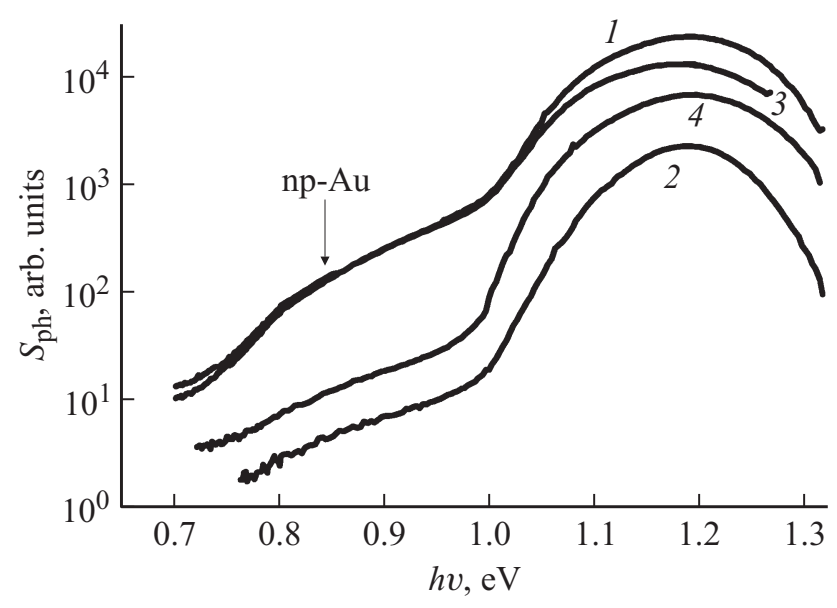

Рис. 5. Спектральные кривые фоточувствительности для МДП-структур $\mathrm{Au} / \mathrm{Zr} / \mathrm{YSZ} / n p-\mathrm{Au} / \mathrm{Si}$ (1), $\mathrm{Au} / \mathrm{Zr} / \mathrm{YSZ} / \mathrm{Si}$ (2), $\mathrm{Au} / \mathrm{Zr} / \mathrm{SiO}_{x} / n p-\mathrm{Au} / \mathrm{Si}(3)$ и $\mathrm{Au} / \mathrm{Zr} / \mathrm{SiO}_{x} / \mathrm{Si}(4)$.

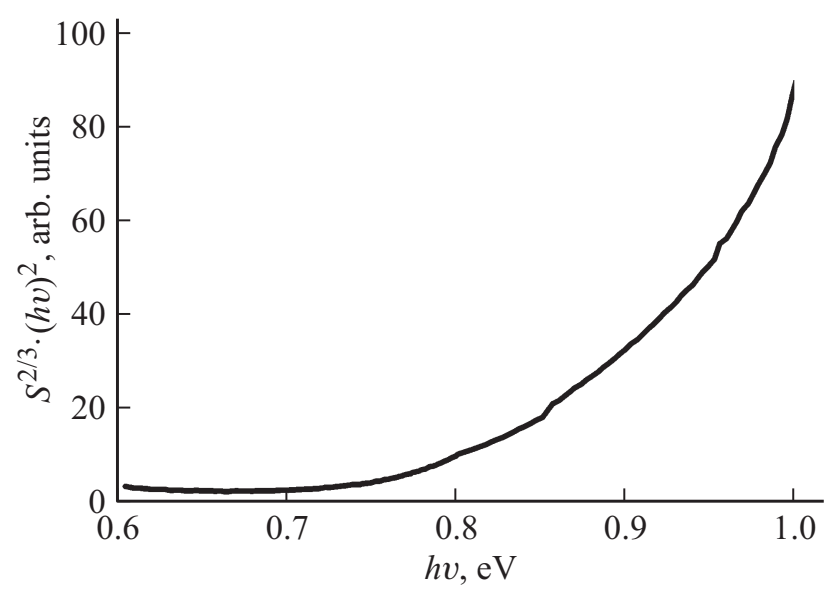

Рис. 6. Кривая спектральной чувствительности, построенная в координатах Луковского для МДП-структуры $\mathrm{Au} / \mathrm{Zr} / \mathrm{YSZ} / n p-\mathrm{Au} / \mathrm{Si}$.
На рис. 5 показаны спектральные кривые фоточувствительности для МДП-структур $\mathrm{Au} / \mathrm{Zr} / \mathrm{YSZ} /$ $n p-\mathrm{Au} / \mathrm{Si}(1), \mathrm{Au} / \mathrm{Zr} / \mathrm{YSZ} / \mathrm{Si}(2), \mathrm{Au} / \mathrm{Zr} / \mathrm{SiO}_{x} / n p-\mathrm{Au} / \mathrm{Si}$ (3) и $\mathrm{Au} / \mathrm{Zr} / \mathrm{SiO}_{x} / \mathrm{Si}(4)$.

На спектральных кривых фоточувствительности структур с np-Аu была обнаружена примесная полоса с максимумом вблизи 0.85 эВ (рис. 5, кривые 1 и 3). Фотоотклик в этой области увеличивался на $1-2$ порядка величины по сравнению с фотооткликом от структур без наночастиц (рис. 5, кривые 2 и 4). Анализ области фоточувствительности, созданной $n p$-Аu, методом Луковского [15] не выявил отчетливых дискретных уровней. На кривой Луковского (рис. 6) не было обнаружено ярко выраженных прямолинейных участков, экстраполяцией которых до пересечения с осью энергий можно было определить точное энергетическое положение дискретных уровней, близких к потолку валентной зоны $\mathrm{Si}$ c энергией $E_{V}$.

Грубая оценка методом экстраполяции спектральных кривых Луковского прямыми линиями дает значения уровней с энергиями от $E_{V}+0.20$ эВ до $E_{V}+0.40$ эВ. Эти уровни заполнены при $U=0$ электронами, и с них возможна фотоэмиссия электронов в $c$-зону $\mathrm{Si}$, которая проявлялась в спектрах фоточувствительности. Обнаруженная область примесной фоточувствительности, связанная с наличием $n p-\mathrm{Au}$, представляет интерес для ряда практических приложений. Следует отметить, что описанное воздействие не соответствует поведению примеси атомарного $\mathrm{Au}$ в объемном $\mathrm{Si}$, которое хорошо изучено и обусловлено двумя дискретными уровнями в запрещенной зоне $\mathrm{Si}$ : акцепторным уровнем с энергией $E_{c}-0.54$ эВ и донорным уровнем с энергией $E_{V}+0.35$ эВ $[16,17]$.

В таблице приведены полученные значения $\varphi_{s}, E_{N}$ и параметры ПС $\left(E_{a}, \sigma_{n}\right.$ и $\left.N_{s s}\right)$ для структур с $n p-\mathrm{Au}$ и без них.

\section{4. Заключение}

Показано, что формирование наночастиц золота на границе раздела диэлектрик/полупроводник в МДПструктурах на основе кремния с YSZ и $\mathrm{SiO}_{2}$ в качестве диэлектрика оказывало существенное влияние на свойства этих структур. Это влияние было выражено в инвертировании поверхности $\mathrm{Si}$, увеличении плотности поверхностных состояний, сдвиге уровня закрепления 
уровня Ферми на поверхности $\mathrm{Si}$ (точки электронейтральности) к потолку валентной зоны кремния и возникновении области примесной фоточувствительности конденсаторной фотоэдс с максимумом около 0.85 эВ.

Работа поддержана Министерством образования и науки России в рамках государственного задания № 2014/134 (код проекта 2591) и 3.2441.2014/К.

\section{Список литературы}

[1] О.Н. Горшков, И.Н. Антонов, Д.О. Филатов, М.Е. Шенина, А.П. Касаткин, Д.А. Павлов, А.И. Бобров. Письма в ЖТФ, 42 (1), 72 (2016).

[2] S.K. Ray, S. Maikap, W. Banerjee, S. Das. J. Phys. D: Appl. Phys., 46, 153 001-1 (2013).

[3] M. Uenuma, K. Kawano, B. Zheng, N. Okamoto, M. Horita, S. Yoshii, I. Yamashita, Y. Uraoka. Nanotechnology, 22, 215201 (2011).

[4] W. Guan, S. Long, R. Jia, M. Liua. Appl. Phys. Lett., 91, 062111 (2007).

[5] С.В. Тихов, О.Н. Горшков, И.Н. Антонов, А.П. Касаткин, М.Н. Коряжкина. Письма ЖТФ, 40 (19), 18 (2014).

[6] A.N. Mikhaylov, A.I. Belov, D.V. Guseinov, D.S. Korolev, I.N. Antonov, D.V. Efimovykh, S.V. Tikhov, A.P. Kasatkin, O.N. Gorshkov, D.I. Tetelbaum, A.I. Bobrov, N.V. Malekhonova, D.A. Pavlov, E.G. Gryaznov, A.P. Yatmanov. Mater. Sci. Eng. B, 194, 48 (2015).

[7] С.В. Тихов, О.Н. Горшков, Д.А. Павлов, И.Н. Антонов, А.И. Бобров, А.П. Касаткин, М.Н. Коряжкина, М.Е. Шенина. Письма в ЖТФ, 40 (9), 9 (2014).

[8] В.Н. Овсюк. Электронные процессы в полупроводниках с областями пространственного заряда (Новосибирск, Наука: Сиб. отд-ние, 1984).

[9] С.В. Тихов. ФТП, 29 (4), 742 (1995).

[10] И.А. Карпович, Д.О. Филатов. Фотоэлектрическая диагностика квантово-размерных гетеронаноструктур (Нижний Новгород, Изд-во Нижегородского ГУ, 2010).

[11] В.А. Зуев, А.В. Саченко, И.Б. Толпыго. Неравновесные приповерхностные процессы в полупроводниках и полупроводниковых приборах (М., Сов. радио, 1977).

[12] С.В. Тихов, О.Н. Горшков, А.П. Касаткин, И.Н. Антонов, М.Н. Коряжкина. Тез. докл. ХІХ Междунар. симп. „Нанофизика и наноэлектроника“ (Нижний Новгород, Россия, 2015) т. 2, с. 665.

[13] В.А. Киселев. ФТТ, 31 (12), 142 (1989).

[14] Л.С. Берман, А.А. Лебедев. Емкостная спектроскопия глубоких центров в полупроводниках (Л., Наука: Ленингр. отд-ние, 1981).

[15] G. Lucovsky. Sol. St. Commun., 3, 299 (1965).

[16] К. Рейви. Дефекты и примеси в полупроводниковом кремнии (М., Мир, 1984).

[17] С.3. Зейнабидинов, О.О. Маматкаримова, И.Г. Турсунов, У.А. Туйчиев. ФТП, 34 (6), 641 (2000).

Редактор А.Н. Смирнов

\section{Electric and photoelectric properties of metal-insulator-semiconductor structures based on Si with Au nanoparticles deposited at the insulator/semiconductor boundary}

\author{
M.N. Koryazhkina, S.V. Tikhov, O.N. Gorshkov, \\ A.P. Kasatkin, I.N. Antonov \\ Lobachevsky State University of Nizhny Novgorod, \\ 603950 Nizhny Novgorod, Russia
}

\begin{abstract}
It is shown that the formation of Au nanoparticles at the insulator/silicon interface in the structures with high surface state density leads to the pinning of the Fermi energy in the insulator/silicon interface shifting to the valence band of silicon and to the surface state density increasing at the energies close to the Fermy energy Herewith, the peak on the photosensitivity of capacitor photovoltage occurs at $0.85 \mathrm{eV}$ due to electron photoemission from the $n p$-Au electron states formed near the valence band of silicon.
\end{abstract}

